\title{
Refractory Epilepsy: The Role of Positron Emission Tomography
}

\author{
Tommy L.H. Chan, Jonathan Romsa, David A. Steven, Jorge G. Burneo
}

\begin{abstract}
Rationale: Presurgical localization of the epileptogenic focus is critical to successful surgery. Traditionally, localization of the epileptogenic focus depends on seizure semiology, scalp video-electroencephalography (vEEG), magnetic resonance imaging (MRI), neuropsychological assessment, and, when needed, intracranial EEG (iEEG). We aimed to explore the role of positron emission tomography (PET) in the presurgical evaluation of patients with refractory epilepsy. Methods: A retrospective review was conducted on patients from London Health Sciences Centre (London, Ontario) with refractory epilepsy who underwent PET from September of 2011 to April of 2016. The accuracy of epileptogenic focus localization was compared between different investigative modalities (MRI, vEEG, iEEG, PET), and the outcomes were documented, including seizure freedom after surgical resection, improvement of seizure frequency, guidance for further investigations, and exclusion of patients from further evaluation. Patients who underwent surgery were followed up at 3 months and onward. Results: We identified 62 patients with refractory epilepsy who underwent PET. The mean age was 34 years (range $=20-68)$. A total of 36 had concordant PET and vEEG findings: 6 had surgical resection and either became seizure-free (29.4\%) or had improvement in seizure frequency (5.9\%) at 3 months; 11 had surgical resection and either became seizure-free (29.4\%) or had improvement in seizure frequency $(35.3 \%)$ at 3 months, but required iEEG for final verification. Conclusions: PET has an important role in presurgical evaluation of patients with refractory epilepsy. It may allow resection of the epileptogenic focus without the need for iEEG, guiding intracranial electrode placement for further localization of the epileptogenic focus, or exclusion of patients from further evaluation.
\end{abstract}

RÉSUMÉ: Rôle de la tomographie par émission de positons dans le cas de l'épilepsie réfractaire. Fondement: Les chances de succès d'une opération chirurgicale visant à traiter l'épilepsie réfractaire dépendent en grande partie de la localisation préopératoire du foyer épileptogène. De façon habituelle, on procède à la localisation de ce foyer en s'appuyant sur les éléments suivants : la sémiologie clinique des crises survenues, un électroencéphalogramme effectué à partir du cuir chevelu et couplé à un enregistrement vidéo (EEGv), un examen d'IRM, une évaluation neuropsychologique et, quand cela est nécessaire, un électroencéphalogramme intracrânien (EEGi). Dans cette étude, nous entendons explorer le rôle de la tomographie par émission de positons (TEP) en ce qui a trait à l'évaluation préopératoire de patients atteints d'épilepsie réfractaire. Méthodes: Nous avons mené un examen rétrospectif de patients du Centre des sciences de la santé de London (Ontario) souffrant d'épilepsie réfractaire à qui l'on avait fait passer un examen de TEP entre septembre 2011 et avril 2016. L'exactitude de la localisation des foyers épileptogènes a été comparée au moyen de divers examens (IRM, EEGv, EEGi, TEP). Les résultats de ces examens ont été consignés, ce qui a inclus notamment les périodes sans crises à la suite d'une résection chirurgicale, la diminution de la fréquence des crises mais aussi des indications cliniques en vue d'examens plus approfondis et l'exclusion de patients d'évaluation ultérieure. À noter qu'un suivi des patients ayant subi une intervention chirurgicale était assuré de façon continue à partir du troisième mois. Résultats: Nous avons identifié 62 patients souffrant d'épilepsie réfractaire à qui l'on avait fait passer un examen de TEP. Leur âge moyen était de 34 ans (étendue $=20-68$ ). Un total de 36 patients a montré des résultats concordants lors d'examens de TEP et d'EEGv. De ce nombre, 6 avaient subi une résection chirurgicale et ne souffraient plus de crises (29,4\%) ou avait vu diminuée la fréquence de leurs crises au troisième mois $(5,9 \%) ; 11$ avaient subi une résection chirurgicale et ne souffraient plus de crises $(29,4 \%)$ ou avaient vu diminuée la fréquence de leurs crises au troisième mois $(35,3 \%)$ mais avaient nécessité un examen d'EEGi en vue d'une dernière confirmation. Conclusions: Les examens de TEP peuvent jouer un rôle important dans l'évaluation préopératoire de patients atteints d'épilepsie réfractaire. Dans certains cas, ils permettent la résection des foyers épileptogènes sans qu'il ne soit nécessaire de procéder à un examen d'EEGi, ce qui suppose d'installer des électrodes sur le cuir chevelu permettant une meilleure localisation, ou d'exclure des patients d'examens plus approfondis.

Keywords: Epilepsy, Refractory epilepsy, Epilepsy surgery, Positron emission tomography, PET

doi:10.1017/cjn.2017.244

Can J Neurol Sci. 2018; 45: 30-34

Approximately $30 \%$ of patients with epilepsy have medically refractory seizures. Refractory epilepsy is defined as the failure to achieve sustained seizure freedom despite an adequate trial of two antiepileptic drugs. ${ }^{1}$ In this group of patients, epilepsy surgery has the potential to control seizures if an epileptogenic focus can be localized.

Traditionally, localization of the epileptogenic focus depends on seizure semiology, scalp video electroencephalography (vEEG), magnetic resonance imaging (MRI), and neuropsychological assessment. However, these traditional methods have their limitations. vEEG poorly measures epileptiform activity in certain cortical areas, such as the opercular regions, mesial hemispheres, and the insula, among others. MRI may fail to demonstrate a structural abnormality.

From the Department of Clinical Neurological Sciences, London Health Sciences Centre, London, Ontario, Canada (TLHC, DAS, JGB); the Department of Medical Imaging, London Health Sciences Centre, London, Ontario, Canada (JR); the Epilepsy Program, Schulich School of Medicine, Western University, London, Ontario, Canada (DAS, JGB).

Received December 16, 2016. Final Revisions Submitted May 13, 2017. Date of ACCEPTANCE July 13, 2017.

Correspondence to: Jorge G. Burneo, 339 Windermere Road, London, Ontario, Canada, N6A5A5. Email: jburneo2@uwo.ca 
Seizure semiology may implicate different epileptogenic zones due to overlapping semiology or to propagation phenomena. ${ }^{2}$

In the absence of an identifiable epileptogenic focus derived from traditional methods, intracranial EEG (iEEG) remains the gold standard in seizure localization. This technique is invasive, but, most importantly, it requires a strong hypothesis regarding the seizure focus. ${ }^{3}$ Consequently, advanced neuroimaging techniques have been used to guide the placement of intracranial electrodes and complement other traditional methods in localizing epileptogenic foci. One commonly used adjuvant imaging technique is positron emission tomography (PET). We have been using ${ }^{18}$ F-FDG PET computed tomography (CT) since 2011 as part of our presurgical workup of certain patients being considered for epilepsy surgery. This technique images the topographic distribution of glucose $\left(2-\left[{ }^{18} \mathrm{~F}\right]-\right.$ fluoro-2deoxy-D-glucose [FDG]) uptake in the brain and provides a picture of cerebral metabolism. ${ }^{4}$ PET is typically performed in the interictal state with the goal of detecting focal areas of relative hypometabolism.

The specific roles of this technique have not been standardized, and utilization varies among epilepsy centers. In this paper, we aimed to explore the role of PET CT in presurgical evaluation of patients with refractory epilepsy and to compare the accuracy of PET with standard methods (MRI, vEEG, and semiology) for epileptogenic focus localization.

\section{MeTHODS}

The database of the epilepsy program at the London Health Sciences Centre (London, Ontario) between September of 2011 and April of 2016 was reviewed. We identified 62 patients with refractory epilepsy who underwent PET. Patients who underwent surgery were followed up at 3 months and onward. Findings on 1.5T MRI, vEEG, and iEEG were analyzed and compared to PET. The outcomes were documented, including seizure freedom after surgical resection (Engel classification I), improvement in seizure frequency, guidance for further investigations, and the exclusion of patients from further evaluation. Informed consents were obtained.

We excluded patients who did not have a standard presurgical workup, were lost to follow-up, refused surgical intervention, or are currently awaiting PET imaging. Descriptive statistics were applied.

\section{Results}

\section{Subject Demographics}

We identified 62 patients (33 males and 29 females) with refractory epilepsy who underwent PET. The mean age was 34 years (range $=20-68$ ), and the mean onset of epilepsy was 17 years of age (range $=1-54$ ). Forty-eight patients had temporal lobe epilepsy (TLE) and 14 had extratemporal lobe epilepsy (eTLE).

\section{MRI Findings}

A total of 48 patients had unremarkable MRI imaging, while 14 had abnormal MRI findings: 8 had mesial temporal sclerosis, 2 had cortical dysplasia, 1 had a dysembryoplastic neuroepithelial tumor (DNET), 1 had hippocampal asymmetry, 1 had subcortical tubers, and 1 had a temporal ganglioglioma.

\section{PET Results}

Thirty-six patients had concordant (or matching) findings on PET and vEEG, while 26 had discordant findings on PET and vEEG.
Of the 36 patients with vEEG and PET concordance, 17 (13 TLE, 4 eTLE) underwent surgical resection of the epileptogenic focus (47.2\%), 7 TLE are awaiting surgical resection, 11 (7 TLE, 4 eTLE) were deemed ineligible for surgical resection due to multiple epileptogenic foci identified (an example is shown in Figure 1), and 1 TLE patient with concordant findings is awaiting further assessment with iEEG.

Of the 26 patients with discordant vEEG and PET, 10 (6 TLE, 4 eTLE) had PET studies reported as normal and 16 (14 TLE, 2 eTLE) as abnormal. In those patients whose PET was reported as normal, 3 became controlled on medications, 2 had multiple epileptogenic foci, 1 had surgical resection of the epileptogenic focus following assessment with iEEG, 3 are awaiting further iEEG monitoring, and 1 declined further investigation. Those 16 patients with an abnormal PET either had more than a single area of hypometabolism or the location of the hypometabolic area differed from the potential epileptogenic foci detected with the use of vEEG, MRI, neuropsychology, and/or SPECT and iEEG.

\section{Surgical Outcomes}

Of the 36 patients with concordant PET and vEEG, 17 (13 TLE, 4 eTLE) underwent surgical resection of the epileptogenic focus (Table 1). Six of these patients (5 TLE, 1 eTLE) underwent surgical resection of the epileptogenic focus without iEEG, while 11 (8 TLE and 3 eTLE) underwent the required iEEG.

Of those who had surgery without iEEG (6/17), 5 became seizure-free (Engel I) and 1 had an improvement in seizure frequency at 3 months. An illustrative case is shown in Figure 2. Of those who required iEEG (11/17), 5 patients became seizurefree (Engel I), while 6 had improvement in seizure frequency at 3 months.

Out of the 26 patients with discordant PET and vEEG, 1 had surgical resection of the epileptogenic focus and became seizurefree (with iEEG confirmation) at 3 months, despite having a normal PET. Of the remaining 25 patients, 3 are awaiting further assessment with iEEG, while 22 were not candidates for resective epilepsy surgery.

\section{DISCUSSION}

It has been established that MRI evidence of hippocampal atrophy is a strong predictor of excellent postoperative seizure control after an anterior temporal lobectomy. ${ }^{5}$ However, some individuals with epilepsy do not have an identifiable structural lesion on MRI. In our study population, only $23 \%$ of patients had abnormal findings on MRI. It should be pointed out that the MRI scans performed in this series were all with a $1.5 \mathrm{~T}$ field strength. In one case series, the yield of $1.5 \mathrm{~T}$ was as low as $17 \%$ in detecting lesions in focal epilepsy, ${ }^{6}$ and it is possible that additional lesions might have been identified on 3T MRI that were not seen on the $1.5 \mathrm{~T}$ scans performed here. It is acknowledged that higher-field MRI, particularly 3T, is preferred for epilepsy imaging and may identify subtle abnormalities such as focal cortical dysplasia. ${ }^{7}$ It provides higher-resolution imaging and can increase lesion identification and thus improve outcomes following epilepsy surgery. ${ }^{8}$ The number of abnormal MRI findings also varies among different care settings due to differences in epilepsy imaging protocols. For example, it has been shown that specific epilepsy-protocol MRI scans identified focal lesions in $85 \%$ of patients who previously were reported not to have a lesion with a conventional MRI protocol. ${ }^{9}$ 


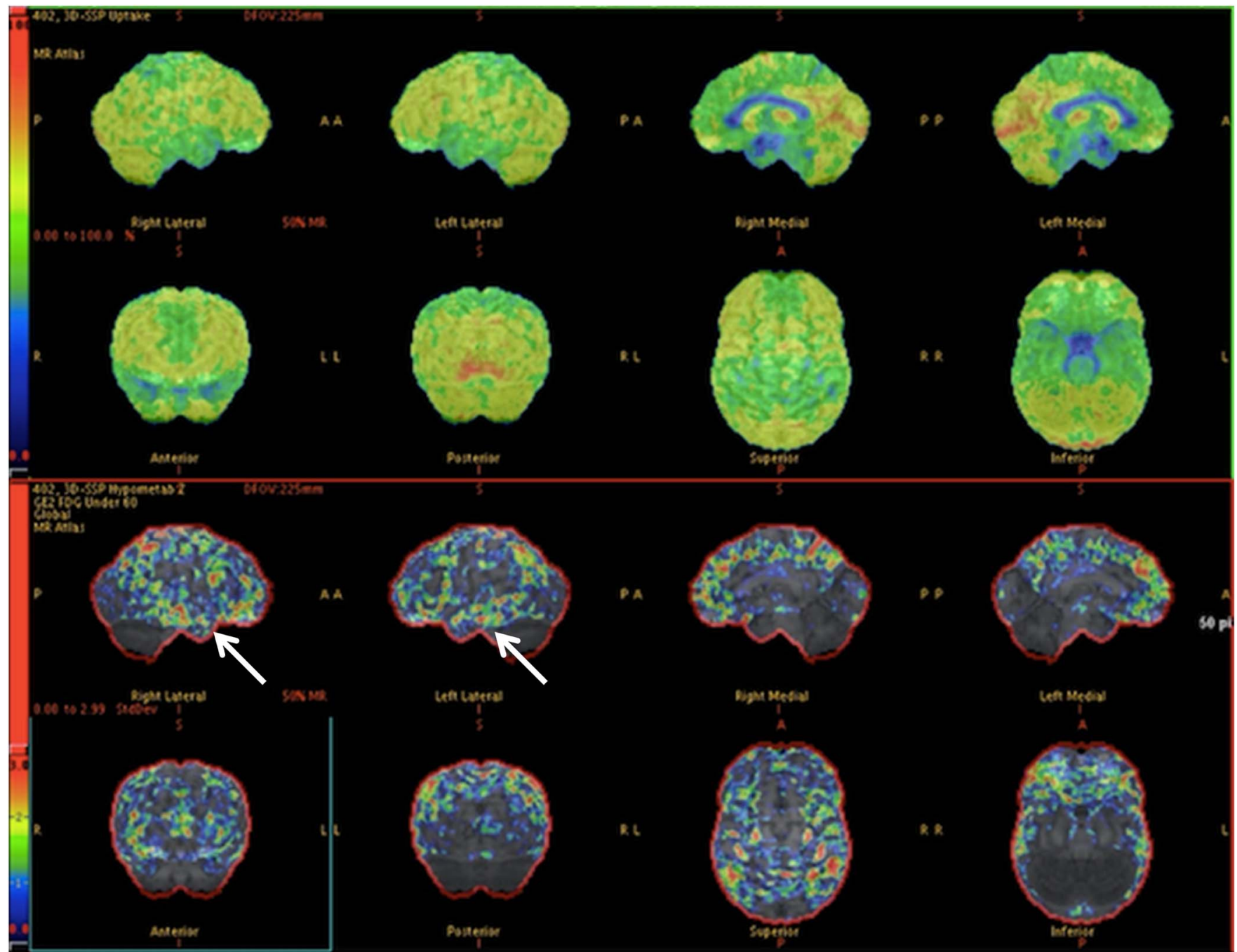

Figure 1: PET images depicting hypometabolism in the mesial aspect of both temporal lobes anteriorly. (Arrows) There is subtle asymmetry, with the right side being slightly more severely affected. The patient (57-year-old male) was deemed ineligible for surgical resection due to bitemporal involvement, which was also found on $v E E G$ (normal MRI).

Regardless of MRI type, when traditional methods are incongruent or no structural lesion can be identified, functional imaging such as PET can play a complementary role in localization of the epileptogenic focus and may minimize the need for invasive EEG monitoring or provide guidance for intracranial electrodes placement. It has been previously mentioned in the literature that lateralizing information identified from PET can guide intracranial electrode placement with results comparable to MRI. ${ }^{10}$
Unfortunately, very few studies have examined the surgical outcome of patients with normal MRI and matching PET/vEEG findings. In a published review article, ${ }^{13}$ primary studies compared PET accuracy with surgical outcome. Overall, the percentage of patients in whom PET correctly localized a seizure focus and had a good surgical outcome ranged from 36 to $89 \%$, and it was higher $(71-89 \%)$ in those with temporal lobe epilepsy. ${ }^{11}$ Our results demonstrate a favorable surgical outcome in patients with

Table 1: Surgical outcome of patients with concordant PET and vEEG (with and without iEEG)

\begin{tabular}{l|c|c|c}
\hline Concordant group* & Seizure-free (no. of patients)^ & Improvement in seizure frequency (no. of patients) & Total number (no. of patients) \\
\hline PET and vEEG (no iEEG performed) & $5(29.4 \%)$ & $1(5.9 \%)$ & $6(35.3 \%)$ \\
\hline PET and vEEG (iEEG performed) & $5(29.4 \%)$ & $6(35.3 \%)$ & $11(64.7)$ \\
\hline Totals & $10(58.8 \%)$ & $7(41.2 \%)$ & 17 \\
\hline
\end{tabular}

*A total of 19 patients were excluded: 11 patients with multiple epileptogenic foci identified (nonsurgical candidates), 1 patient awaiting iEEG for further clarification, and 7 patients awaiting surgical resection (did not undergo iEEG assessment).

^Measured 3 months postoperatively. 


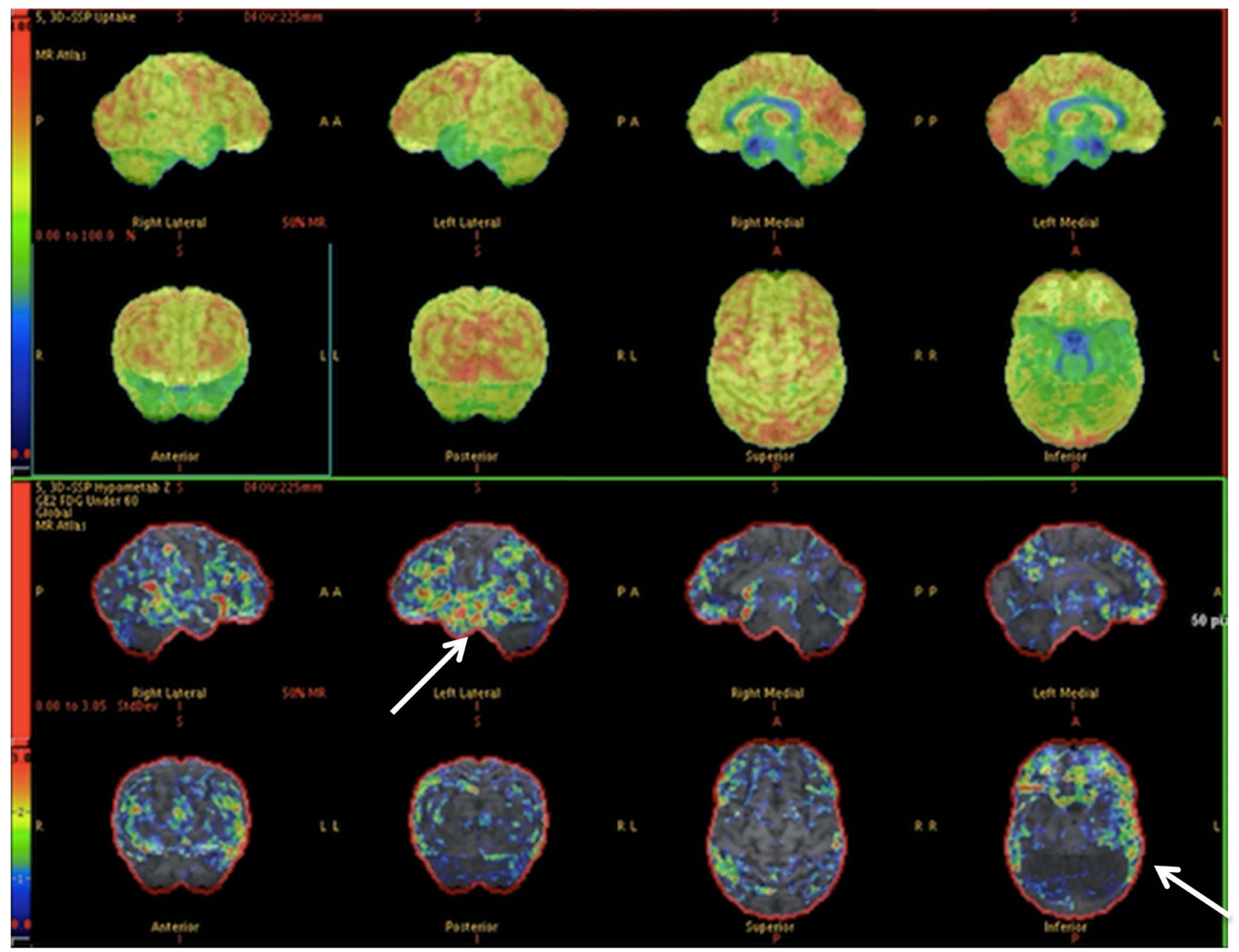

Figure 2: PET images depicting relative left temporal lobe hypometabolism with respect to the right temporal lobe. This was congruent with the $v E E G$. This patient (35-year-old male) had a left temporal lobectomy and became seizure-free (normal MRI).

concordant PET and vEEG findings as illustrated in Table 1, indicating that in selected cases iEEG may not be required prior to successful surgical resection. Patients who are awaiting iEEG and anticipating surgical resection were included in the analysis, as the information provided by PET guided the placement of intracranial electrodes. Based on our experience to date, good surgical outcome is anticipated in patients awaiting surgery with concordant PET and vEEG.

The performance of PET imaging in our population would appear to reflect the heterogeneity of epilepsy in our study population, which included a significant number of extratemporal lobe epilepsy patients. ${ }^{12,13}$ In a systematic review, ${ }^{11}$ PET demonstrated 56-90\% agreement with seizure onset localized by iEEG, and the sensitivity increased to $70-90 \%$ in those with temporal lobe hypometabolism. There is less information available regarding the usefulness of PET in eTLE, but it appears somewhat less sensitive in these cases. ${ }^{14}$ An exception may be patients with cortical dysplasia, in whom the reported sensitivity of PET varies between 60 and $92 \% .{ }^{15}$ The sensitivity increased by $8-23 \%$ when PET results were combined with MRI or EEG. ${ }^{11}$
In our study, 26 patients had discordant PET and EEG results. There are several factors that might explain these findings. First, the area of hypometabolism typically extends beyond the epileptogenic zone, making it less useful for precise neuroanatomic localization. ${ }^{14}$ Second, other non-epilepsy factors can cause regional hypometabolism, such as preexisting parenchymal injury (gliosis and encephalomalacia) from infarcts, trauma, infections, and neurodegeneration (e.g., Alzheimer's disease). Focal seizures that quickly secondarily generalize may have reduced sensitivity. Finally, the epileptogenic focus may be too small to be detected by PET. ${ }^{16}$

The main limitation of our study resides in selection bias, as only potential surgical patients underwent PET. In addition, investigators were not blinded to the results of all the investigations prior to PET reporting. However, these two issues reflect what occurs in "real-life" scenarios, and they do not minimize the value of PET.

Finally, FDG was the PET tracer used in this study. The use of other tracers (e.g., 11C-flumazenil, $\left[{ }^{18} \mathrm{~F}\right]$ fluoroethyl-L-tyrosine, $\alpha$-methyltryptophan, and serotonin agonists) is under investigation and may improve the sensitivity and specificity of PET for presurgical localization. $^{17}$ 
In summary, PET has an important role to play in presurgical evaluation of patients with refractory epilepsy. In selected cases, it may allow resection of the epileptogenic focus without the need for iEEG. PET may also help guide intracranial electrode placement for further localization of the epileptogenic focus. In addition, it may aid in detection of multiple epileptogenic foci, thereby identifying patients as ineligible for surgical resection, and thus avoiding the need for invasive intracranial electrode studies.

\section{Disclosures}

Jorge G. Burneo reports personal fees from UCB Canada for speaker bureau work and grants from UCB Canada, outside the submitted work.

David A. Steven, Tommy L.H. Chan, and Jonathan Romsa have no conflicts of interest to disclose.

\section{REFERENCES}

1. Kwan P, Arzimanoglou A, Berg AT, et al. Definition of drug resistant epilepsy: consensus proposal by the ad hoc Task Force of the ILAE Commission on Therapeutic Strategies. Epilepsia. 2010; 51(6):1069-77.

2. Tufenkjian K, Lüders HO. Seizure semiology: its value and limitations in localizing the epileptogenic zone. J Clin Neurol. 2012;8(4):243-50.

3. Spencer SS. The relative contributions of MRI, SPECT, and PET imaging in epilepsy. Epilepsia. 1994;35(Suppl 6):S72-89.

4. Desai A, Bekelis K, Thadani VM, et al. Interictal PET and ictal subtraction SPECT: sensitivity in the detection of seizure foci in patients with medically intractable epilepsy. Epilepsia. 2013;54(2):341-50.

5. Kuzniecky R, Burgard S, Faught E, Morawetz R, Bartolucci A. Predictive value of magnetic resonance imaging in temporal lobe epilepsy surgery. Arch Neurol. 1993;50(1):65-9.
6. King MA, Newton MR, Jackson GD, et al. Epileptology of the first-seizure presentation: a clinical, electroencephalographic, and magnetic resonance imaging study of 300 consecutive patients. Lancet. 1998;352(9133):1007-11.

7. Martinez-Rios C, McAndrews MP, Logan W, Krings T, Lee D, Widjaja E. MRI in the evaluation of localization-related epilepsy. J Magn Reson Imaging. 2016;44(1):12-22.

8. Rubinger L, Chan C, D'Arco F, et al. Change in presurgical diagnostic imaging evaluation affects subsequent pediatric epilepsy surgery outcome. Epilepsia. 2016;57(1):32-40.

9. Von Oertzen J, Urbach $\mathrm{H}$, Jungbluth $\mathrm{S}$, et al. Standard magnetic resonance imaging is inadequate for patients with refractory focal epilepsy. J Neurol Neurosurg Psychiatry. 2002; 73(6):643-7.

10. Knowlton RC, Elgavish RA, Bartolucci A, et al. Functional imaging, II: prediction of epilepsy surgery outcome. Ann Neurol. 2008; 64(1):35-41.

11. Burneo JG, Poon R, Kellett S, Snead OC. The utility of positron emission tomography in epilepsy. Can J Neurol Sci. 2015;42(6):360-71.

12. Henry TR, Engel J, Mazziotta JC. Clinical evaluation of interictal fluorine-18-fluorodeoxyglucose PET in partial epilepsy. J Nucl Med. 1993;34(11):1892-8.

13. Van Paesschen W, Dupont P, Sunaert S, Goffin K, Van Laere K. The use of SPECT and PET in routine clinical practice in epilepsy. Curr Opin Neurol. 2007;20(2):194-202.

14. Knowlton RC. The role of FDG-PET, ictal SPECT, and MEG in the epilepsy surgery evaluation. Epilepsy Behav. 2006;8(1):91-101.

15. Lerner JT, Salamon N, Hauptman JS, et al. Assessment and surgical outcomes for mild type I and severe type II cortical dysplasia: a critical review and the UCLA experience. Epilepsia. 2009; 50(6):1310-35.

16. Goffin K, Dedeurwaerdere S, Van Laere K, Van Paesschen W. Neuronuclear assessment of patients with epilepsy. Semin Nucl Med. 2008;38(4):227-39.

17. Fedi M, Reutens D, Okazawa H, et al. Localizing value of alpha-methylL-tryptophan PET in intractable epilepsy of neocortical origin. Neurology. 2001;57(9):1629-36. 